Umstand, dass er nicht in die früheren Auseinandersetzungen involviert war, auch eine gewisse Unbefangenheit, was seine in diesem Werk erarbeiteten Urteile umso wertvoller macht.

Wiegand hat eine große Geschichte der direkten Demokratie in Deutschland geschrieben, ein grundlegendes Werk für jede weitere Beschäftigung mit der Materie. Die Spezialliteratur hat daneben immer noch ihr Recht.

Otmar Jung

\title{
Direkte Demokratie in Schweizer Städten: empirische Bestandsaufnahme
}

Bützer, Michael: Direkte Demokratie in Schweizer Städten. Ursprung, Ausgestaltung und Gebrauch im Vergleich (Reihe „Studien zur Sachunmittelbaren Demokratie“, Band 4), Nomos Verlagsgesellschaft, Baden-Baden 2007, 308 Seiten, $€ 49,-$.

Dass Recht und Praxis unmittelbarer Demokratie gemeinhin vor allem für die übergemeindlichen Ebenen behandelt werden, ist kein Alleinstellungsmerkmal der Schweiz. Allerdings kommt dort hinzu, dass sich die einschlägigen Rechtsgrundlagen nicht nur von Kanton zu Kanton, sondern auch unter den jeweiligen (Einwohner-)Gemeinden maßgeblich unterscheiden. Vor diesem Hintergrund gibt die Arbeit von Michael Bützer, die 2006 von der Philosophischen Fakultät der Universität Zürich als Dissertation angenommen wurde, hinsichtlich der 118 (geographischen) Städte mit mehr als 10.000 Einwohnern umfassend Auskunft. Weitgehend ausgeklammert bleiben Formen der Versammlungsdemokratie, wobei aber aufgezeigt wird, dass die durchschnittliche Teilnahme an Bürgerentscheiden deutlich höher liegt als diejenige an Versammlungen. Der Autor bilanziert deshalb, Volksabstimmungen stellten „ganz eindeutig geeignetere Instrumente als Bürgerversammlungen dar, um die demokratische Qualität von kommunalpolitischen Entscheiden zu gewährleisten" (S. 71).

Bützers Untersuchung gliedert sich in zwei Teile: Zunächst werden die politischen Institutionen, vor allem die bestehenden Einrichtungen direkter Demokratie, und anschließend der jeweilige Abstimmungsgebrauch analysiert. Dabei bezieht sich die Arbeit in beiden Teilen auf die Jahre 1990 bis 2000.

Im ersten Teil folgen auf einen Überblick zu den kommunalverfassungsrechtlichen Rahmenbedingungen Angaben zu Verbreitung und Ausgestaltungen von obligatorischen und fakultativen Referenden, Initiativen und Gegenvorschlägen in den einzelnen Städten. Diese werden durch Kurzbeschreibungen zu den Rechtslagen in den Kantonen ergänzt, die im Anhang abgedruckt sind. Dabei stellt der Autor die Materie umfassend dar und analysiert Entwicklungstendenzen während des Untersuchungszeitraums. Er fragt auch nach dem direktdemokratischen Öffnungsgrad der politischen Systeme. Diesen macht er zum einen an der materiellen Reichweite der Volksrechte, zum anderen an ihren formellen Erfordernissen fest. Hierbei mahnt er wegen der vereinfachten Zusammenhänge zwar zur Vorsicht, doch kann immerhin ein recht deutlicher Zusammenhang zwischen Öffnungsgraden und Rechtstraditionen aufgezeigt werden. Bützer unterscheidet hier zwischen deutschschweizer Gemeinden mit Versammlungssystem (Landgemeinden) beziehungsweise Stadtgemeinden 
und Orten mit französischer Tradition. Im Ergebnis ist der Öffnungsgrad Deutschschweizer Landgemeinden am höchsten, der französischer Gemeinden am geringsten, wobei für die Untersuchungszeit eine leichte Annäherung festgestellt wird.

Der zweite Teil stützt sich auf Daten des Instituts für direkte Demokratie der Universität Genf, wo der Autor als Forschungsassistent tätig war. Ergebnis eines Vergleichs der Abstimmungshäufigkeit in Bund, Kantonen und Städten ist, dass die Abstimmungstätigkeit im Bund mit Abstand am höchsten und in den Städten durchschnittlich am geringsten ist. Nur dort hat sie sich während der neunziger Jahre auch stetig verringert. Während im Bund Initiative, fakultatives und obligatorisches Referendum in etwa gleich häufig waren, liegt auf den anderen Ebenen das obligatorische vor dem fakultativen Referendum und der Initiative klar an erster Stelle. In den Städten führte es zu etwa sechsmal mehr Abstimmungen als jede andere Einrichtung direkter Demokratie. Dennoch hat sich die Anzahl obligatorischer Referenden während des Untersuchungszeitraums um nahezu ein Drittel verringert, was mit Blick auf die Finanzentscheide plausibel erklärt werden kann.

Zuletzt fragt Bützer nach den Gründen für das Auftreten von Gemeindebürgerabstimmungen, die er anhand verschiedener Modelle untersucht. Er kann unter anderem zeigen, dass die gesamte Abstimmungstätigkeit durch die rechtstraditionelle Zugehörigkeit einer Stadt und unabhängig davon durch ihren direktdemokratischen Öffnungsgrad bestimmt wird. Zudem erhöhte sich der Abstimmungsgebrauch bei steigender sozialer Komplexität, während wirtschaftliche Schwierigkeiten (überraschenderweise) zu niedrigeren Anwendungszahlen führten. Ein statistisch signifikanter Einfluss von Unterschriftenhürden auf die Praxis konnte nicht nachgewiesen werden.

Für die geleistete Arbeit, insbesondere für die erhobenen und systematisch dargestellten Daten gebührt dem Autor Anerkennung. Die Untersuchung füllt eine Lücke. Das gilt für die Rechtsgrundlagen und ihre Anwendungspraxis gleichermaßen. Die Ausführungen sind stets gut lesbar, vor allem weil der Verfasser trotz seiner detaillierten Erhebungen nicht den Blick für übergeordnete Fragestellungen verliert. Im Gegenteil wäre teilweise die Schilderung einzelner Anwendungsfälle interessant. Die Erklärungen hinsichtlich der Befunde dürften für die wissenschaftliche und politische Auseinandersetzung über die Schweiz hinaus von Bedeutung sein, wenn auch ein Vergleich wegen der strukturellen Unterschiede nur eingeschränkt möglich ist. In Anbetracht der Bedeutung kleinerer Gemeinden würde man sich auch zu ihnen entsprechende Untersuchungen wünschen; diese sind aber aufgrund der hohen Gemeindezahl allenfalls stichprobenartig möglich. 